\section{Case Reports in Oncology}

\title{
Large Skin Ulcer and Delayed Wound Healing around a Colostomy in a Patient with Metastatic Colorectal Cancer Receiving Vascular Endothelial Growth Factor Receptor-2 Inhibitor Therapy
}

\author{
Koichi Taira ${ }^{a}$ Yuji Nadatani ${ }^{a}$ Shinji Hirano ${ }^{a} \quad K^{2}$ iyoshi Maeda ${ }^{b}$ \\ Yasuhiro Fujiwara ${ }^{a}$ \\ aDepartment of Gastroenterology, Osaka City University Graduate School of Medicine, \\ Osaka City, Japan; ${ }^{b}$ Department of Surgical Oncology, Osaka City University Graduate \\ School of Medicine, Osaka City, Japan
}

\section{Keywords}

Colostomy · Delay wound healing · Metastatic colorectal cancer $\cdot$ Skin ulcer $\cdot$ Vascular endothelial growth factor receptor-2 inhibitor

\begin{abstract}
Ramucirumab is an antiangiogenic agent targeting vascular endothelial growth factor receptor (VEGF)-2 that has been approved for second-line treatment of patients with metastatic colorectal cancer. VEGF-targeted therapy has various distinctive adverse effects owing to its antitumour effects. However, little is known with regard to its skin toxicity, such as its ability to cause skin ulcers. We report a case of large skin ulceration around a colostomy and delayed healing caused by ramucirumab. A 58-year-old patient diagnosed with rectal cancer with liver and lung metastases. He was administered folinic acid, fluorouracil (5-FU), and oxaliplatin (FOLFOX) and bevacizumab as first-line treatment. A laparoscopic colostomy was performed for suspected worsening of the bowel obstruction. He was then administered folinic acid, 5 fluorouracil, and irinotecan (FOLFIRI) and ramucirumab as second-line treatment after surgery. However, dehiscence and a small skin ulceration caused by ramucirumab developed around the colostomy which increased in size and became necrotic; therefore, he was administered
\end{abstract}


only FOLFIRI, without ramucirumab. The ulcer decreased in size slightly with surgical debridement and showering. He resumed FOLFIRI and ramucirumab.

\section{Introduction}

Vascular endothelial growth factor (VEGF)-targeted therapy has been used widely for several types of solid tumors such as metastatic colon cancer, non-squamous non-small-cell lung cancer, and human epidermal growth factor receptor (HER2) 2-negative breast cancer [1-3]. In colorectal cancer, the use of VEGF-targeted therapy in combination with chemotherapy is the accepted standard of treatment. Bevacizumab, ramucirumab, and aflibercept are VEGF-targeted therapy agents approved for use in Japan. These drugs were approved by the United States Food and Drug Administration (FDA) for the treatment of metastatic colorectal cancer (mCRC) in 2008, 2014, and 2011, respectively.

Ramucirumab is a human immunoglobulin G subclass 1 (IgG1) monoclonal antibody that specifically binds to the extracellular domain of VEGFR-2 with high affinity, preventing the binding of the VEGF-A, C, and D ligands and receptor activation [4]. In a pivotal randomized, double-blind, placebo-controlled phase III trial (RAISE) [5], the safety and efficacy of ramucirumab in combination with folinic acid, 5 fluorouracil, and irinotecan (FOLFIRI) as secondline therapy in patients with $\mathrm{mCRC}$ that progressed during or after first-line therapy with bevacizumab, oxaliplatin, and a fluoropyrimidine were proved. Ramucirumab carries a specific adverse reaction profile as well as other VEGF-targeted therapy, including hypertension, bleeding, gastrointestinal perforation, impaired wound healing, and arterial thromboembolism. However, skin toxicity such as skin ulcers from VEGF-targeted therapy is rarely reported. Moreover, there are no such reports on ramucirumab.

In this report, we describe a case of a large skin ulceration around a colostomy and delayed healing caused by ramucirumab. To our knowledge, this is the first case report detailing the clinical course associated with such skin toxicity around a colostomy following VEGF-targeted therapy.

\section{Case Presentation}

In June 2016, a 58-year-old male patient was referred to our hospital for further investigation and treatment of mCRC. He had no notable medical history, such as a history of diabetes. A thoracoabdominal contrast-enhanced computed tomography (CT) scanning showed thickening of the rectal wall and multiple metastatic liver and lung lesions (Fig. 1). Colonoscopy revealed a type-2 lesion with an associated ulcer centered on the rectum, with severe stenosis, beyond which the colonoscope could not pass. Histopathological examination of the biopsy specimens showed adenocarcinoma with RAS mutation. We could not evaluate for BRAF gene mutations at that time.

In June 2016, first-line treatment comprising folinic acid, fluorouracil (5-FU), and oxaliplatin (FOLFOX) and bevacizumab was initiated. He initially achieved a partial response after two cycles of treatment, with no major toxicities. In August, after six cycles, computed tomography imaging revealed stable disease; however, his constipation had worsened and he was diagnosed with subileus. Surgical treatment was planned to avoid ileus. The patient received one dose of FOLFOX without bevacizumab to prevent impaired surgical wound healing. He 
underwent a laparoscopic colostomy in September, 19 and 33 days after the final chemotherapy and bevacizumab treatments, respectively.

Second-line therapy with folinic acid, 5 fluorouracil, and irinotecan (FOLFIRI) and ramucirumab was commenced in October, 23 days after surgery. Although severe adverse events did not occur, a small dehiscence of the mucocutaneous junction and a small skin ulceration developed around the colostomy on November 1, 2016 after day 5 of the second cycle (Fig. $2 \mathrm{a}$ ), which progressively increased in size and became necrotic. We diagnosed the skin ulcer as an adverse effect of ramucirumab and stopped ramucirumab administration on November 25 (Fig. 2b). The patient received only FOLFIRI, without ramucirumab, for the next three cycles. He underwent surgical debridement three times and showered twice a day, washing the ulcer around the colostomy. After reaching its maximum size on December 5 at day 11 of the first cycle of FOLFIRI without ramucirumab (Fig. 2c), the ulcer slowly decreased in size and regenerating epithelium appeared. On January 20, 2017 (Fig. 2d), about 2 months after the ramucirumab was stopped, therapy with FOLFIRI and ramucirumab was resumed. At day 15 after treatment resumption, he presented as an outpatient at our Emergency Department for anorexia and abdominal pain. CT imaging showed intraperitoneal free air due to perforation of the site of dehiscence of the mucocutaneous junction of the colostomy. He was managed non-operatively and treated with antibiotics because the peritonitis was not severe. He survived this event with non-operative management and began third-line treatment 20 days later.

\section{Discussion}

VEGF-targeted therapy is widely used for the treatment of metastatic cancer. Several studies have demonstrated that VEGF-targeted therapy in combination with various first-line and second-line chemotherapies significantly improves overall and progression-free survival. Clinical benefit has been derived from agents that bind to circulating VEGF, a key factor in vasculogenesis and angiogenesis [6]. VEGF is expressed in almost all organs and plays a fundamental physiological role [7]. These agents are generally well-tolerated, but by inhibiting VEGF, they have distinctive adverse effects, such as skin toxicity.

Skin toxicity is caused by blocked of vasodilation, increased vascular permeability, and angiogenesis [8]. The timing of VEGF-targeted therapy initiation and cessation warrants careful consideration because although skin toxicity is rare it is potentially life-threatening. Although there are a few reports on the skin toxicity of bevacizumab, there is no such report on ramucirumab, which is the most recently approved VEGF-targeted therapy.

Bose et al. reported impaired wound healing at a rate of $1.3 \%$ for adjuvant bevacizumab administered 28-60 days after surgery [9]. Cessation of bevacizumab is recommended at least 5 weeks before a surgical intervention, with treatment resumption no less than 4 weeks later because of the 21-day half-life of bevacizumab. The NSABP C-08 trial in the adjuvant setting for stage II or III colorectal cancer showed a skin toxicity rate of $1.7 \%$ in the bevacizumab group, with a median time to occurrence of 5 months [10]. The risk of skin toxicity increased even if it was initiated 6 weeks from the time of surgery and continued up to 6 months after its cessation. However, the half-life of ramucirumab was about 8 days. In our case, surgical intervention was performed 33 days after the final bevacizumab treatment and 21 days before the first ramucirumab treatment. Therefore, we believed that there would be no problem in our case, given the sufficient washout period around the operation. We could have stopped 
the treatment for a longer period; however, it was difficult to decide when to resume VEGFtargeted therapy.

The complication of necrosis of skin metastases has been reported in other cancers as well. Cottu et al. reported 12 cases of breast cancer with extensive skin involvement that were treated with bevacizumab [11]. Nine patients who had presented with erosive skin lesions developed deep ulcerations and necrosis, with large areas of skin layers lost and delayed healing of the surgical flaps, despite early discontinuation of bevacizumab. The average time to wound healing was 24 weeks (range 4-72, median 20). Considering the therapeutic effect of VEGF-targeted therapy, it is difficult to stop treatment for periods as long as 24 weeks. Therefore, it is important to be aware of this specific toxicity and treat it as soon as possible. By responding quickly to small erosions, the period of treatment interruption can be shortened without the progression to ulcers.

\section{Conclusion}

We report a case of a skin ulcer and delayed wound healing around a colostomy in a patient with $\mathrm{mCRC}$ receiving VEGF-targeted therapy.

Our case shows that careful attention must be paid when using VEGF-targeted therapy for cancer in patients with a colostomy. In the cases of appearance of small ulcers, immediate cessation of VEGF-targeted therapy should be considered and close care is important.

\section{Statement of Ethics}

Written informed consent was obtained from the patient for publication of this case report and any accompanying images. A copy of the written consent is available for review by the Editor of this journal.

\section{Disclosure Statement}

The authors have no conflicts of interest to declare.

\section{Author Contributions}

KT and YN participated in the treatment of the patient, drafting, and revision of the manuscript. SH and KM participated in the treatment of the patient. MO and YF participated in the analysis and interpretation of the data, as well as in the drafting and revision of the manuscript. All authors read and approved the final manuscript.

\section{References}

1 Saltz LB, Clarke S, Díaz-Rubio E, Scheithauer W, Figer A, Wong R, et al. Bevacizumab in combination with oxaliplatin-based chemotherapy as first-line therapy in metastatic colorectal cancer: a randomized phase III study. J Clin Oncol. 2008 Apr;26(12):2013-9.

2 Sandler A, Gray R, Perry MC, Brahmer J, Schiller JH, Dowlati A, et al. Paclitaxel-carboplatin alone or with bevacizumab for non-small-cell lung cancer. N Engl J Med. 2006 Dec;355(24):2542-50. 
3 Miller K, Wang M, Gralow J, Dickler M, Cobleigh M, Perez EA, et al. Paclitaxel plus bevacizumab versus paclitaxel alone for metastatic breast cancer. N Engl J Med. 2007 Dec;357(26):2666-76.

4 Spratlin JL, Cohen RB, Eadens M, Gore L, Camidge DR, Diab S, et al. Phase I pharmacologic and biologic study of ramucirumab (IMC-1121B), a fully human immunoglobulin G1 monoclonal antibody targeting the vascular endothelial growth factor receptor-2. J Clin Oncol. 2010 Feb;28(5):780-7.

5 Tabernero J, Yoshino T, Cohn AL, Obermannova R, Bodoky G, Garcia-Carbonero R, et al.; RAISE Study Investigators. Ramucirumab versus placebo in combination with second-line FOLFIRI in patients with metastatic colorectal carcinoma that progressed during or after first-line therapy with bevacizumab, oxaliplatin, and a fluoropyrimidine (RAISE): a randomised, double-blind, multicentre, phase 3 study. Lancet Oncol. 2015 May;16(5):499-508.

6 Kim KJ, Li B, Winer J, Armanini M, Gillett N, Phillips HS, et al. Inhibition of vascular endothelial growth factorinduced angiogenesis suppresses tumour growth in vivo. Nature. 1993 Apr;362(6423):841-4.

7 Chen HX, Cleck JN. Adverse effects of anticancer agents that target the VEGF pathway. Nat Rev Clin Oncol. 2009 Aug;6(8):465-77.

8 Bates DO, Jones RO. The role of vascular endothelial growth factor in wound healing. Int J Low Extrem Wounds. 2003 Jun;2(2):107-20.

9 Bose D, Meric-Bernstam F, Hofstetter W, Reardon DA, Flaherty KT, Ellis LM. Vascular endothelial growth factor targeted therapy in the perioperative setting: implications for patient care. Lancet Oncol. 2010 Apr;11(4):373-82.

10 Allegra CJ, Yothers G, O'Connell MJ, Sharif S, Colangelo LH, Lopa SH, et al. Initial safety report of NSABP C-08: A randomized phase III study of modified FOLFOX6 with or without bevacizumab for the adjuvant treatment of patients with stage II or III colon cancer. J Clin Oncol. 2009 Jul;27(20):3385-90.

11 Cottu PH, Fourchotte V, Vincent-Salomon A, Kriegel I, Fromantin I. Necrosis in breast cancer patients with skin metastases receiving bevacizumab-based therapy. J Wound Care. 2011 Sep;20(9):403-4.

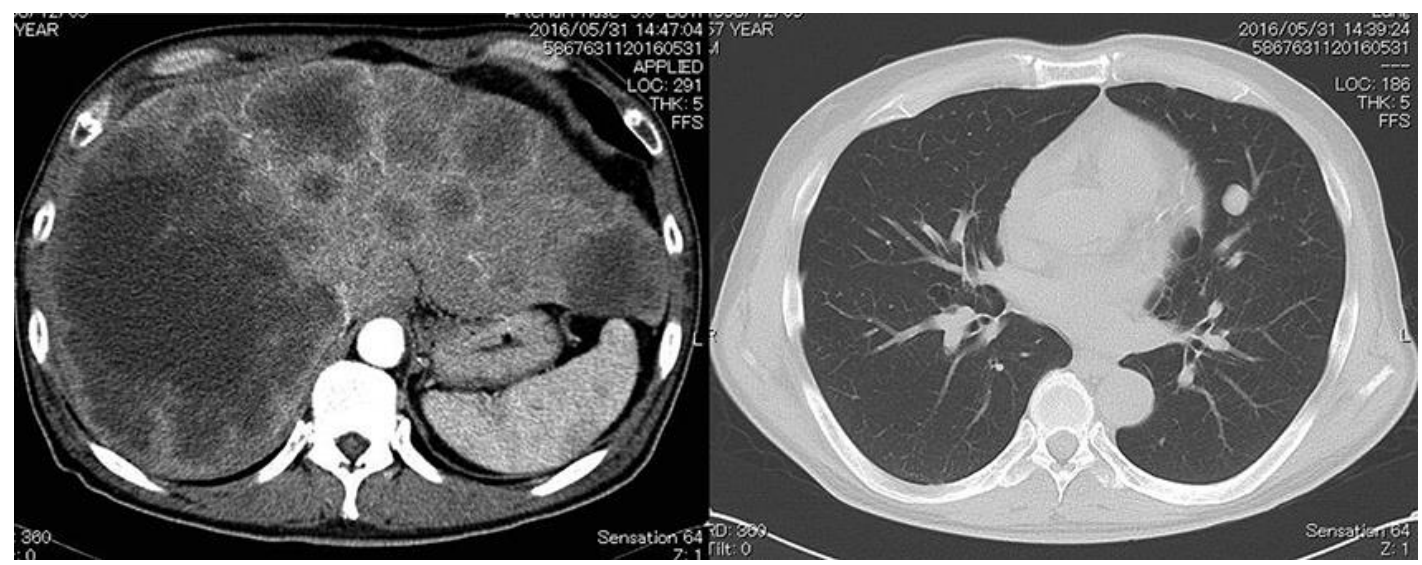

Fig. 1. CT scanning showed multiple metastatic liver and lung lesions before chemotherapy. 


\section{Case Reports in Oncology}
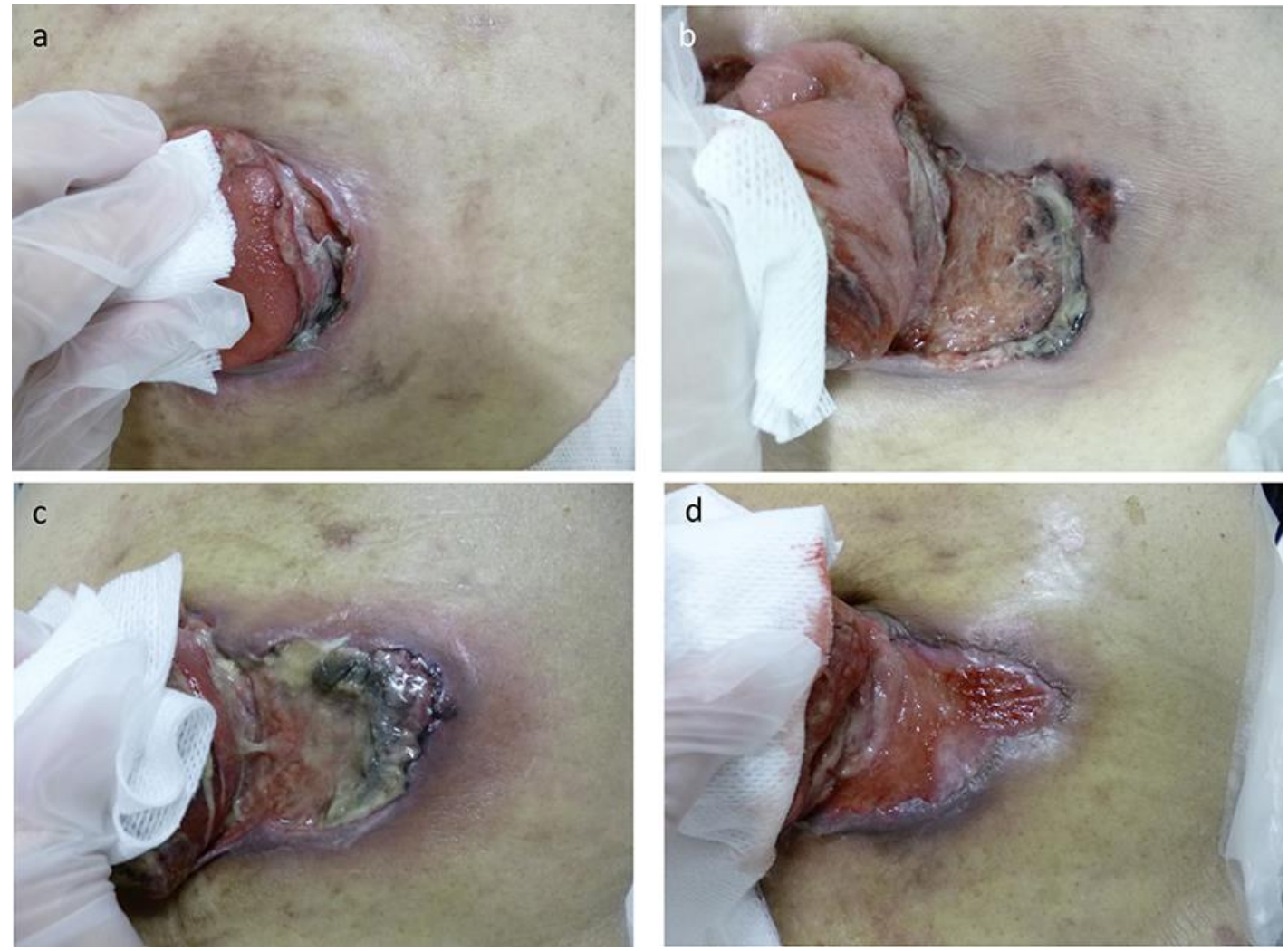

Fig. 2. (a) Skin ulcer around a colostomy on November 1, 2016 after day 5 of the second cycle of FOLFIRI and ramucirumab. A small dehiscence of the mucocutaneous junction and a small skin ulceration developed around the colostomy. (b) Skin ulcer around a colostomy on November 25, 2016 after day 14 of the third cycle of FOLFIRI and ramucirumab. The ulcer got larger day by day and had necrosis. (c) Skin ulcer around a colostomy on December 5, 2016 at day 11 of the first cycle of FOLFIRI without ramucirumab. The ulcer reached maximum size in the course. (d) Skin ulcer around a colostomy on January 20, 2017 at day 1 of the first cycle of FOLFIRI and ramucirumab. The ulcer slowly decreased in size and regenerating epithelium appeared. 